\title{
GALLILEI COVARIANT QUANTUM MECHANICS IN ELECTROMAGNETIC FIELDS
}

\author{
H. E. WILHELM \\ Michelson Laboratory, Physics Division, Naval Weapons Center \\ China Lake, California 93555, L.S.A.
}

(Received July 18, 1984)

\begin{abstract}
A formulation of the quantum mechanics of charged particles in time-dependent electromagnetic fields is presented, in which both the Schroedinger equation and wave equations for the electromagnetic potentials are Galilei covariant. It is shown that the Galilean relativity principle leads to the introduction of the electromagnetic substratum in which the matter and electromagnetic waves propagate. The electromagnetic substratum effects are quantitatively significant for quantum mechanics in reference frames, in which the substratum velocity $w$ is in magnitude comparable with the velocity of light $c$. The electromagnetic substratum velocity $w$ occurs explicitly in the wave equations for the electromagnetic potentials but not in the Schroedinger equation.
\end{abstract}

KEY WORDS AND PHRASES Quantum mechantcs, electromagnetic fields and substratum, Galilet covariance

1980 MATHEMATICS SUB.JECT CLASSIFIC.ITION CODE 8ID25, 81C40. 78125: 78A40

\section{INTRODUCTION}

One of the most interesting basic equations of physics is that of Schroedinger [1] for the matter waves $\Psi(\mathbf{r}, t)$. His so-called "wave equation" is not of hyperbolic type, but a parabolic equation, which propagates the $\Psi$-waves with infinite speed, $c=\infty$ (Wilhelm and Hong [2] ). The Schroedinger equation yields energy eigenvalues for quasi-stationary quantum systems $\left(i \hbar \partial_{t}=\hbar \omega\right)$, which are in agreement with the experimental data, except for fine-structure details which require consideration of the electron spin (Barut [3] ) Only very recently, the Schroedinger equation has been applied to proper (non-oscillatory) time-dependent quantum systems (Wilhelm and Hong [2]; Husumi [4]; Stutz and Schlitt [5]; Wilhelm [6]). The Schroedinger equation is known to be Galilei covariant in the absence of electromagnetic potentials. On the other hand, the general transformation properties of the matter waves $\Psi(\mathbf{r}, t)$ of charged particles in electromagnetic fields $\mathbf{E}(\mathbf{r}, t)$ and $\mathbf{B}(\mathbf{r}, \mathbf{t})$ have apparently not yet been discussed.

The Klein-Gordon and Dirac equations indicate that the $\Psi$-waves propagate with a finite speed $c<\infty$, which is identical with the characteristic speed of electromagnetic wave propagation, $c=(\mu \varepsilon)^{\frac{1}{2}} \simeq$ $3 \times 10^{8} \mathrm{~m} / \mathrm{sec}$. That matter waves $\Psi(r, t)$ and electromagnetic waves $E(r, t), B(r, t)$ propagate with the same wave speed $c$ is physically not surprising, since matter appears to be another form of electromagnetic energy (Poincaré [7]; Hansenoehrl [8]) The experimentally confirmed mass-energy relation $\Delta \mathrm{E}=\Delta \mathrm{mc}^{2}$ gives direct evidence for the electromagnetic nature of matter Indeed, the physical derivations of $\Delta \mathrm{E}=\Delta \mathrm{mc}^{2}$ by Poincaré $[7 \mid$ and $\mathrm{H}$ asenoehrl [8] are based on the electromagnetic energy hypothesis of matter 
The following transformation theory is concerned with quantum mechanics for charged particles in electromagnetic fields, both in the wave-mechanical formulation of Schroedinger $[1]$ and the hydrodynamic formulation of Madelung [9]. We demonstrate that the principle of the covariance of the laws of physics with respect to Galilei transformations, requires an electromagnetic substratum, in which the matter and electromagnetic waves propagate. Whereas the velocity field $\mathbf{w}$ of the substratum appears explicitly in the electromagnetic wave equations, the substratum velocity $w$ drops out of the wave and hydrodynamic equations of quantum mechanics in Galilei transformations This explains the "elusiveness" of the electromagnetic substratum in the hasic equations of quantum mechanics Significant electromagnetic substratum effects exist in inertial frames with large substratum drifts, $|w| \lesssim c, e . g$, in reference frames comoving with relativistic electron beams

The discovery of the isotropic microwave radiation of $2.7^{\circ} \mathrm{K}$ in the universe by Wilson and Penzias [10] has shown that Maxwell, Heaviside, Lorentz, Poincaré, Michelson, and Sagnac were essentially correct in assuming that Maxwell's equations hold for a universe in which the carrier of the electromagnetic waves (EM substratum) is at rest According to Mansour and Sexl [11], the thermal microwave background is cosmologically singled out as a preferred frame of reference, "and the existence of this electromagnetic substratum system is in perfect agreement with all experimental evidence." The theory of Bagge |12| explains mass inertia as an interaction with the electromagnetic substratum, and gives a physıcal derivation of relativistic dynamics. By means of Bagge's [13] generalized commutation relations for quantum systems with a minimum length $q_{0}$ and time $t_{0}=q_{0} / c$ and the electromagnetic substratum concept, Winterberg $|1+|$ has developed a nonlinear relativity theory which eliminates the singularities of quantum electrodynamics on a physical basıs

The reaction $\mathrm{e}^{-}+\mathrm{e} \leftrightarrows 2 \mathrm{y}$ observed by Anderson [15] indicates that positrons, electrons, and photons are excitations of the electromagnetic substratum The electron-positron substratum was first predicted by Dirac [16] from his relativistic wave equation. Relativistic quantum mechanics led also to the introduction of a zero point vacuum energy density with the Lorentz invariant $\omega^{3}$-spectrum, so that the energy density of the electromagnetic substratum would be infinite (Power [17]) As demonstrated by Landau and Lifshitz |18|, the requirement of causality in the special theory of relativity gives rise to the postulate that elementary particles must be point-like. Since in physical reality an infinite vacuum energy and point-like particles do not exist, present thinking is that gravity must provide a natural cutoff at the Planck length $L_{P} \sim\left(\hbar G / c^{3}\right)^{\frac{1}{2}} \sim 10^{15} \mathrm{~m}$ so that the mass density of the electromagnetic substratum would have the (absurd) value $\rho_{w} \sim h / L_{p} p^{4} \sim$ $10^{93} \mathrm{~kg} / \mathrm{m}^{3}$ proposed by Wheeler [19].

It is obvious that the previously developed theories of the electron-positron vacuum (Dirac [16]; Power [17]; Landau and Lifshitz [18], Wheeler [19] ) or electromagnetic substratum are physically unsatisfying since they are based on the special theory of relativity, which holds only for the interactions of point particles (with infinite electromagnetic field energy and self-acceleration) and divergent zero point energy spectra $\mathrm{dE} / \mathrm{d} \omega \sim \omega^{3}$ (Landau and Lifshitz [18]) A promısing way out of the present difficulties of relativistic physics has been shown in the generalized quantum mechanics of Bagge $|13|$ and the non-Einsteinian relativity theory of Winterberg [14]. The latter gives for the electromagnetic substratum a spectral energy density of the form (Winterberg [14])

$$
\mathrm{dE} / \mathrm{d} \omega=\left(\hbar / 2 \pi^{2} \mathrm{c}^{3}\right) \omega^{3} \exp \left(-\omega \mathrm{t}_{0}\right)
$$

Thus, the total zero point vacuum energy is finite, $E<\infty\left[\right.$ for $t_{0} \equiv 0$, Eq. (1 1 1) reduces to the special relativity spectrum $\propto \omega^{3}$ with divergent energy, $\left.E=\infty\right]$. At high energies $\hbar \omega$, this spectral energy density is compensated by a negative pressure resulting from the conform-invariant Winterberg $[20]$ metric 
A microscopic theory of the electromagnetic substratum is not required for the understanding of the derivation of the covariant quantum mechanical and electromagnetic field equations for inertial frames with substratum flow to be presented. We will make use only of the hypothesis of the existence of the electromagnetic substratum, which appears to be sufficiently supported by experimental and theoretical investigations (Wilson [10]: Mansouri and Sexl [11]. Bagge [12]; Winterberg [14]. Dirac |16|: Power |17]). In concluding this overview, Einstein [21] shall be quoted who foresaw the importance of the electromagnetic substratum for the further development of physics in 1921. "Space without ether is unthinkable, for in such space there not only would be no propagation of light, but also no possibility of existence for standards of space and tıme (measuring rods and clocks), nor therefore any space-tıme in a physical sense."

\section{WAVE-MECHANICAL FORMULATION}

The Schroedinger equation does not contain explicitly the velocity field of the substratum in which the matter waves propagate and is, therefore, certainly applicable in an inertial frame $2^{\circ}\left(\mathbf{r}^{2}, t^{2}, \mathbf{w}^{\circ}=0\right)$, in which the velocity field $w^{\prime}$ of the substratum vanıshes. The parabolic wave equation for the wave function $\psi^{\prime}\left(r^{\circ}, t^{\circ}\right)$ of a particle of mass $m_{0}$, and charge $e_{0} \gtrless 0$ (Galilei invariants) in an electromagnetic field with scalar potential $\phi^{3}\left(\mathbf{r}^{\circ}, t^{\circ}\right)$ and vector potential $\mathbf{A}^{\circ}\left(\mathbf{r}^{\circ}, t^{\circ}\right)$ is for the inertial frame $\Sigma^{\circ}\left(\mathbf{r}^{\circ}, t^{\circ}, \mathbf{w}^{\circ}=0\right)$ (Landau and Lifshitz [1\})

$$
\mathrm{i} \hbar \partial \Psi^{\circ} / \partial \mathrm{t}^{\circ}=\left[\left(-\mathrm{i} \hbar \nabla^{\circ}-e_{1} \mathrm{~A}^{\circ}\right)^{2} / 2 \mathrm{~m}_{0}+\mathrm{e}_{0} \phi^{\circ}+\mathrm{L}^{\circ} \mid \Psi^{\circ}\right.
$$

where

$$
\begin{aligned}
& \left(\mu^{\circ} \varepsilon^{\circ} \partial^{2} / \partial t^{\circ 2}-\nabla^{\circ 2}\right) \mathbf{A}^{\circ}=\mu^{\circ} \mathbf{j}_{\mathrm{e}}^{\circ}, \\
& \left(\mu^{\circ} \varepsilon^{\circ} \partial^{2} / \partial t^{\circ 2}-\nabla^{\circ 2}\right) \phi^{\circ}=\rho_{e}^{\circ} / \varepsilon^{\circ},
\end{aligned}
$$

and

$$
\begin{gathered}
\boldsymbol{\nabla}^{\circ} \cdot \mathbf{A}^{\circ}=-\mu^{\circ} \varepsilon^{\circ} \partial \phi^{\circ} / \partial t^{\circ}, \\
\mathbf{E}^{\circ}=-\nabla^{\circ} \phi^{\circ}-\partial \mathbf{A}^{\circ} / \partial t^{\circ}, \quad \mathbf{B}^{\circ}=\nabla^{\circ} \times \mathbf{A}^{\circ}
\end{gathered}
$$

For reasons of generality, a conservative, non-electromagnetic potential $\mathrm{U}^{\circ}\left(\mathbf{r}^{\circ}, \mathrm{t}^{\circ}\right)(\mathrm{e} \mathrm{g}$, gravitational or oscillator potential) also is included in the Schroedinger equation (2.1). Equations (22) and (2 3) are the electromagnetic wave equations, which determine $A^{\circ}\left(r^{\circ}, t^{\circ}\right)$ and $\phi\left(r^{\circ} t^{\circ}\right)$ from given current density $j_{e}{ }^{\circ}\left(r^{\circ}, t^{\circ}\right)$ and charge density $\mathrm{Pe}^{\circ}\left(\mathrm{r}^{\circ}, \mathrm{t}^{\circ}\right)$ distributions [sources of the electromagnetic field in Eq. (2 5)). Equation (2.4) is the Lorentz gauge which defines the sources of $\mathbf{A}^{\circ}\left(\mathbf{r}^{\circ}, t^{\circ}\right)$, and $\mu^{\circ}, \varepsilon^{\circ}$ are the magnetic and electric permeabilities of "free" space in $\mathbf{\Sigma}^{\circ}$

The Schroedinger equation (2 1) is Galilei covariant, but the electromagnetic wave equations (2 2) and (2.3) are not Galilei covariant The inconsistent simultaneous use of such field equations has been generally accepted not only in nonrelativistic quantum mechanics (Landau and Lifshitz [1] ), but also in classical electrodynamics (Stratton [22] ) and plasma physics (Akhiezer et al [23]), which are based on Galilei covariant equations (Ohm's law, magnetohydrodynamic equations. Boltzmann equations) and the Lorentz covariant Maxwell equations. In order to resolve this inconsistency problem in quantum mechanics, Eqs. (2.1) - (2.5) are subjected first to the Galilei transformation from the inertial frame $\Sigma^{\circ}\left(\mathbf{r}^{\circ}, \mathrm{t}, \mathbf{w}^{\circ}=0\right)$ to the inertial frame $\Sigma(r, t, w)$, which moves with an arbitrary constant velocity $u$ relative to $\Sigma^{\circ}\left(\right.$ let $0^{\circ}=0$ for $\left.t^{\circ}=t=0, F i g 1\right)$.

$$
\begin{gathered}
r=\mathbf{r}^{\circ}-\mathbf{u} \mathrm{t}^{\circ}, \quad \mathrm{t}=\mathrm{t}^{\circ} \\
\partial / \partial \mathrm{t}^{\circ}=\partial / \partial \mathrm{t}-\mathbf{u} \cdot \boldsymbol{\nabla}, \quad \boldsymbol{\nabla}^{\circ}=\boldsymbol{\nabla}
\end{gathered}
$$

where

$$
\begin{gathered}
\Psi^{\circ}=\Psi \exp \left[\mathrm{im}_{0} \mathbf{u} \cdot\left(\mathbf{r}-\mathbf{r}_{0}\right) / \hbar+\mathrm{i}_{2}^{1} \mathrm{~m}_{0} \mathbf{u}^{\prime} \mathrm{t} / \hbar\right], \\
\phi^{\circ}=\phi+\mathbf{u} \cdot \mathbf{A}, \quad \mathbf{A}^{\circ}=\mathbf{A}, \\
\mathbf{j}_{\mathrm{e}}{ }^{\circ}=\mathrm{j}_{\mathrm{e}}+\rho_{\mathrm{e}} \mathbf{u}, \quad \rho_{\mathrm{e}}{ }^{\circ}=\rho_{\mathrm{e}},
\end{gathered}
$$




$$
\mathrm{L}^{\circ}=\mathrm{L}^{-}, \mathbf{w}=-\mathbf{u},
$$

and

$$
\varepsilon^{\circ}=\varepsilon, \mu^{\circ}=\mu
$$

are the corresponding Galilei field transformations and the invariants of free space, respectively The electromagnetic transformations in Eq. (2.9) are known in the form $\mathbf{E}^{\circ}=\mathbf{E}-\mathbf{u} \times \mathbf{B}$ and $\mathbf{B}^{\circ}=\mathbf{B}$ (Stratton [22]), whereas those in Eq (2 10) are obvious. The conservative potential in Eq. (2 11) depends on the distance between-ource and field point (and eventually on time), and is therefore Galilei invariant

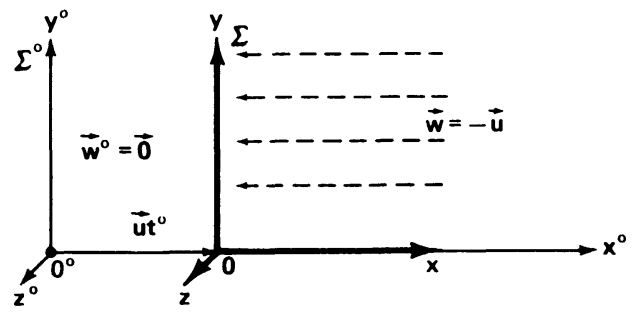

Fig 1. Galilei transformation from inertial frame $\Sigma^{\circ}$ with resting substratum to an inertial frame $\boldsymbol{\Sigma}$ with streaming substratum $\mathbf{w}=-\mathbf{u}$, where $\Sigma$ moves relative to $\Sigma^{\circ}$ with velocity $\mathbf{u}\left(0^{\circ}=0\right.$ for $\left.\mathrm{t}^{\circ}=\mathrm{t}=0\right)$.

In order to derive the Galilei transformation $(28)$ for the Schroedinger field, the complex wave functions in $\boldsymbol{\Sigma}^{\circ}$ and $\boldsymbol{\Sigma}$ are represented in terms of real amplitudes and phases,

$$
\Psi^{\circ}\left(\mathbf{r}^{\circ}, t^{\circ}\right)=R^{\circ}\left(\mathbf{r}^{\circ}, t^{\circ}\right) \mathbf{e}^{t S^{\circ}\left(\mathbf{r}^{\circ}, t^{\circ}\right)}, \Psi(\mathbf{r}, t)=R(\mathbf{r}, t) \mathbf{e}^{(S(r, t)},
$$

where

$$
\begin{array}{cc}
\rho^{\circ}=\mathrm{R}^{\circ 2}, & \mathrm{~m}_{0} \mathbf{v}^{\circ}+\mathrm{e}_{0} \mathbf{A}^{\circ}=\hbar \nabla \cdot \mathrm{S}^{\circ}, \\
\rho=\mathrm{R}^{2}, & \mathrm{~m}_{0} \mathbf{v}+\mathrm{e}_{0} \mathbf{A}=\hbar \nabla \mathrm{S} .
\end{array}
$$

defines their probability densities and velocity fields. Since $\mathbf{v}^{\circ}=\mathbf{v}+\mathbf{u}$ in the Galilei transformation $\Sigma^{\supset} \rightarrow \mathbf{\Sigma}$, Eqs. (2.14) and (2.15) yield

$$
\left(\hbar / \mathrm{m}_{0}\right) \nabla^{\circ} \mathrm{S}^{\circ}-\left(\mathrm{e}_{0} / \mathrm{m}_{0}\right) \mathbf{A}^{\circ}=\left(\hbar / \mathrm{m}_{0}\right) \nabla \mathrm{S}-\left(\mathbf{e}_{0} / \mathrm{m}_{0}\right) \mathbf{A}+\mathbf{u}
$$

or

$$
S_{0}\left(\mathbf{r}^{\circ}, t^{\circ}\right)=S(r, t)+m_{0} \mathbf{u} \cdot\left(\mathbf{r}-\mathbf{r}_{0}\right) / \hbar+f(t)
$$

by integration from $r_{0}$ to $\mathbf{r}\left(r_{0}^{\circ}\right.$ to $\left.r^{\circ}\right)$, since $A^{\circ}=A$ by Eq. (2.9). Combining Eqs. (2.13) and (2.17) results in the transformation (2.8) for the wave function since $\rho^{\circ}=\rho\left(R^{\circ}=R\right)$, where the integration "constant" in Eq (2 17) has been determined as $f(t)=\frac{1}{2} m_{0} u^{2} t / \hbar$ by means of the condition that the Schroedinger equation (2.1) be Galilei covariant. The wave phase in Eqs $(217)$ and $(28)$ is determined up to a constant $C_{0}=-m_{0} u \cdot r_{0} / \hbar \geq 0$

Since the substratum is by definition at rest in $\Sigma^{\circ}$, the substratum streams with velocity $\mathbf{w}=-\mathbf{u}$ in $\Sigma$ (Fig. 1). Substitution of the Galılei transformations (2.6) - (2 11) into Eqs (2 1) - (2.5) yields, therefore, the Schroedinger and electromagnetic equations in the inertial frame with substratum flow, $\mathbf{\Sigma}(\mathbf{r}, t, \mathbf{w})$

$$
\mathrm{i} \hbar \partial \Psi / \partial \mathrm{t}=\left[\left(-\mathrm{i} \hbar \boldsymbol{\nabla}-\mathrm{e}_{0} \mathbf{A}\right)^{2} / 2 \mathrm{~m}_{0}+\mathrm{e}_{\circ} \phi+\mathrm{U}\right] \Psi
$$

where

$$
\begin{aligned}
& {\left[\mu \varepsilon(\partial / \partial t+\mathbf{w} \cdot \nabla)^{2}-\nabla^{2}\right] \mathbf{A}=\mu\left(j_{\mathrm{e}}-\rho_{\mathrm{e}} \mathbf{w}\right),} \\
& {\left[\mu \varepsilon(\partial / \partial \mathrm{t}+\mathbf{w} \cdot \boldsymbol{\nabla})^{2}-\nabla^{2} \mid(\phi-\mathbf{w} \cdot \mathbf{A})=\rho_{\mathrm{e}} / \varepsilon,\right.}
\end{aligned}
$$

and

$$
\begin{aligned}
& \boldsymbol{\nabla} \cdot \mathbf{A}=-\mu \varepsilon(\partial / \partial \mathrm{t}+\mathbf{w} \cdot \boldsymbol{\nabla})(\phi-\mathbf{w} \cdot \mathbf{A}), \\
& \mathbf{E}=-\boldsymbol{\nabla} \phi-\partial \mathbf{A} / \partial \mathrm{t}, \quad \mathbf{B}=\boldsymbol{\nabla} \times \mathbf{A}
\end{aligned}
$$


Equations (2.1) and (2 18) are of the same form, i e, the Schroedinger equation (2 1) is Galilei covariant However, Eqs (2.2) - (25) and Eqs. (2.19) - (2 22) are not of the same form, i.e., the electromagnetic equations $122)-(2.5)$ are not Galilei covariant. According to Maxwell, Heaviside, Hertz, Lorentz, and Poincaré, the electromagnetic equations (2 2) - (2.5) hold only in an inertial frame $\Sigma^{\circ}\left(\mathbf{r}^{\circ}, t^{\circ}, \mathbf{w}^{\circ}=0\right)$, in which the carrier of the electromagnetic fields is at rest (Whittaker [24]). For the latter restriction, the electromagnetic equations (2.2) - (2.5) are not generally valid and cannot, in principle, be Galilei covariant.

()n the other hand, the Schroedinger and electromagnetic equations (2 18) - (222) refer to an inertial frame $\Sigma(\mathbf{r}, t, \mathbf{w})$, in which the substratum flows with an arbitrary constant velocity $\mathbf{w}=-\mathbf{u}($ Fig 1$)$, since $\boldsymbol{\Sigma}$ moves relative to $\Sigma^{\circ}$ with an arbitrary constant velocity $|\mathbf{u}|<c$. For this reason, the electromagnetic field equations (2 19) - (2.22) with substratum flow w could be Galilei covariant in principle, and this is indeed the case

In order to demonstrate that the generalized Schroedinger and electromagnetic field equations (2 18) (2 22) satisfy the Galilean relativity principle, these equations are subjected to the Galilei transformation from the inertial frame $\Sigma(\mathbf{r}, \mathrm{t}, \mathbf{w})$ to the inertial frame $\Sigma^{\prime}\left(\mathbf{r}^{\prime}, \mathrm{t}^{\prime}, \mathbf{w}^{\prime}\right)$, where $\boldsymbol{\Sigma}^{\prime}$ moves relative to $\boldsymbol{\Sigma}$ with the arbitrary constant velocity $u$ (let $0=0^{\prime}$ for $t=t^{\prime}=0$, Fig 2 ).

$$
\begin{gathered}
\mathbf{r}^{\prime}=\mathbf{r}-\mathbf{u t}, \quad \mathrm{t}^{\prime}=\mathrm{t}, \\
\partial / \partial \mathrm{t}=\partial / \partial \mathrm{t}^{\prime}-\boldsymbol{u} \cdot \boldsymbol{\nabla}^{\prime}, \quad \boldsymbol{\nabla}=\boldsymbol{\nabla}^{\prime}
\end{gathered}
$$

where

$$
\begin{gathered}
\Psi=\Psi^{\prime} \exp \left[i m_{0} u \cdot\left(\mathbf{r}^{\prime}-r_{0}\right) / \hbar+i \frac{1}{2} m_{0} u^{\prime} t^{\prime} / \hbar\right] \\
\phi=\phi^{\prime}+u \cdot A^{\prime}, \quad A=A^{\prime} \\
j_{e}=j_{e}^{\prime}+\rho_{e}^{\prime} u, \quad \rho_{e}=\rho_{e}^{\prime} \\
\mathcal{L}=L^{\prime \prime}, \quad w^{\prime}=w-u
\end{gathered}
$$

and

$$
\varepsilon=\varepsilon^{\prime}, \mu=\mu^{\prime}
$$

are the corresponding Galilei field transformations and the invariants of free space, respectively. Substitution of Eqs. (2.23) - (2.29) into Eqs. (2.18) - (2.22) results in the Schroedinger and electromagnetic field equations for the inertial frame $\Sigma^{\prime}\left(\mathbf{r}^{\prime}, t^{\prime}, \mathbf{w}^{\prime}\right)$ :

$$
i \hbar \partial \Psi^{\prime} / \partial t^{\prime}=\left[\left(-i \hbar \nabla^{\prime}-e_{0} A^{\prime}\right)^{2} / 2 m_{0}+e_{0} \Phi^{\prime}+U^{\prime} l \Psi^{\prime}\right.
$$

where

$$
\begin{gathered}
{\left[\mu^{\prime} \varepsilon^{\prime}\left(\partial / \partial t^{\prime}+w^{\prime} \cdot \nabla^{\prime}\right)^{2}-\nabla^{\prime 2}\right] \mathbf{A}^{\prime}=\mu^{\prime}\left(j_{e}{ }^{\prime}-\rho_{e}{ }^{\prime} w^{\prime}\right),} \\
{\left[\mu^{\prime} \varepsilon^{\prime}\left(\partial / \partial t^{\prime}+w^{\prime} \cdot \nabla^{\prime}\right)^{2}-\nabla^{\prime 2}\right]\left(\Phi^{\prime}-w^{\prime} \cdot A^{\prime}\right)=\rho_{e}^{\prime} / \varepsilon^{\prime},}
\end{gathered}
$$

and

$$
\begin{aligned}
& \nabla^{\prime} \cdot \mathbf{A}^{\prime}=-\mu^{\prime} \varepsilon^{\prime}\left(\partial / \partial t^{\prime}+\mathbf{w}^{\prime} \cdot \nabla^{\prime}\right)\left(\Phi^{\prime}-\mathbf{w}^{\prime} \cdot \mathbf{A}^{\prime}\right), \\
& \mathbf{E}^{\prime}=-\nabla^{\prime} \Phi^{\prime}-\partial \mathbf{A}^{\prime} / \partial \mathrm{t}^{\prime}, \quad \mathbf{B}^{\prime}=\boldsymbol{\nabla}^{\prime} \times \mathbf{A}^{\prime}
\end{aligned}
$$

Comparison indicates that Eqs. $(230)-(2.34)$ for the inertial frame $\Sigma^{\prime}\left(\mathbf{r}^{\prime}, t^{\prime}, w^{\prime}\right)$ are of the same form as Eqs $(2.18)-(2.22)$ for the inertial frame $\Sigma(r, t, w)$ Thus. the Galilei covariance of the Schroedinger and electromagnetic field equations $(218)-(2.22)$ with substratum flow $\mathbf{w}$ is demonstrated. This covariance is a necessary condition for Eqs. (2.18) - (2.22) to be generally valid (Galilean relativity principle) The Eqs. (2.18) (2.22) reduce to the usual field equations of nonrelativistic quantum mechanics for $w \rightarrow 0$ 


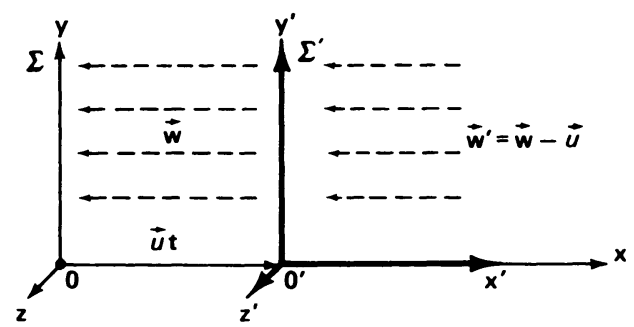

Fig 2: Galilei transformation from inertial frame $\Sigma$ to inertial frame $\Sigma^{\prime}$ where $\Sigma^{\prime}$ moves relative to $\Sigma$ with velocity $u\left(0=0^{\prime}\right.$ for $\left.t=t^{\prime}=0\right)$.

The coupled quantum mechanic/electromagnetic system of field equations $(2.18)-(222)$ holds for all inertial frames $\Sigma(\mathbf{r}, t, \mathbf{w})$, in which the substratum flows with uniform but otherwise arbitrary velocity $|\mathbf{w}|<c$. In addition, Eqs. (2.18) - (222) are approximately correct for arbitrary inertial frames $\Sigma(r, t, w)$ in which the substratum flow field is inhomogeneous, $w=w(r, t)$, if the spatial and temporal nonuniformities of $w(r, t)$ have characteristic extensions $|\Delta \mathbf{r}|$ in space and $|\Delta t|$ in time, which are large in comparison with the dimension and duration of the quantum-electromagnetic process under consideration. The experimental evidence indicates that the latter conditions are satisfied for terrestrial regions of space (Wilson [10]; Mansouri and Sexl [11]).

The fundamental system of wave-mechanical field equations $(2.18)-(2.22)$ indicates that the substratum flow $w$ influences quantum phenomena through its effect on the electromagnetic potentials $(A, \phi)$ in the Schroedinger equation (2 18). Since $\mu \varepsilon=c^{-2}$ in Eqs. $(219)-(220)$, substratum effects are quantitatively significant in reference frames with large drift velocities $|\mathbf{w}| \lesssim \mathbf{c}$

In the hydrodynamic formulation of quantum mechanics (Madelung [91), the Galilean nature of quantum phenomena is most obvious. For this reason, the Galilei covariance of the hydrodynamic equations of quantum mechanics (Wilhelm [25]) is discussed for comparison

\section{HYDRODYNAMIC FORMULATION}

The hydrodynamic formulation of quantum mechanics is due to Madelung [9], and is also known as nonlinear wave mechanics (Wilhelm [25]). Since the Schroedinger equation (2.18) is a complex equation, it can be decomposed into two coupled nonlinear equations for the amplitude $R(r, t)$ and phase $S(r, t)$ of the wave function $\Psi(r, t)$ in Eq. (2.13). Thus, one finds from Eq. (2.18) the nonlinear hydrodynamic equations for the probability density $\rho(r, t)$ and velocity field $v(r, t)$ of a charged particle $\left(e_{0}, m_{0}\right)$ in electromagnetic $(\mathbf{E}, B)$ and conservative ( $-\nabla L)$ force fields:

$$
\begin{aligned}
& \mathrm{m}_{0}(\partial / \partial \mathrm{t}+\mathbf{v} \cdot \boldsymbol{\nabla}) \mathbf{v}=-\boldsymbol{\nabla}\left[\mathrm{U}-\left(\hbar^{2} / 2 \mathrm{~m}_{0}\right)\left(\nabla^{2} \rho^{\frac{t}{t}}\right) / \rho^{\frac{1}{t}}\right]+\mathrm{e}_{0}(\mathbf{E}+\mathbf{v} \times \mathbf{B}), \\
&(\partial / \partial \mathrm{t}+\mathbf{v} \cdot \boldsymbol{\nabla}) \rho=-\rho \nabla \cdot \mathbf{v},
\end{aligned}
$$

where

$$
\rho=\Psi \bar{\Psi}, \quad \mathbf{v}=\left(\hbar / \mathrm{m}_{1}\right) \nabla(\operatorname{lm} \ln \Psi)-\left(\mathbf{e}_{0} / \mathrm{m}_{0}\right) \mathbf{A}
$$

in accordance with Eq $(215)$ The fields $\mathbf{E}(\mathbf{r}, \mathrm{t})$ and $\mathbf{B}(\mathbf{r}, \mathrm{t})$ are determined by the electromagnetic equations with iubstratum flow w already stated in Eqs. (2 19) - (2.22)

The nonlinear quantum dynamic equations (3.1) and (3.2) differ from the hydrodynamic equations of an Ideal, isobaric $(-\nabla p=0)$ gas of charged particles through the normalization of the density field $\rho(r, t)$ and the quantum or Madelung $[9 \mid$ potential,

$$
Q=-\left(\hbar^{2} / 2 m_{0}\right)\left(\nabla^{2} \rho^{\frac{1}{2}}\right) / \rho^{\frac{1}{2}}
$$


Furthermore, the canonical momentum field $m_{0} \mathbf{v}+e_{0} \mathbf{A}$ of the charged particle in the electromagnetic field is quantized,

$$
\oint\left(\mathrm{m}_{0} \mathbf{v}+\mathbf{e}_{0} \mathbf{A}\right) \cdot \mathrm{d} \mathbf{r}=\hbar \oint \mathrm{d} S=\hbar 2 \mathrm{rm}, \quad \mathrm{m}=0, \pm 1, \pm 2, . \pm \infty
$$

since the wave function $\Psi(r, t)$ is invariant with respect to a change of its phase $S(r, t)$ by an integer multiple $m$ of $2 \pi$

In this regard, it is remarkable that the nonlinear equations of classical hydrodynamics and magnetohydrodynamics can be simplified and condensed mathematically into complex wave equations by means of the inverse transformation (Wilhelm $[26,27]$ ). These are, however, nonlinear since they contain nonlinear pressure and self-consistent electromagnetic potentials (Wilhelm $[26,271$ ).

The hydrodynamic quantum equations $(31)$ and (3.2) and their auxiliary electromagnetic equations (2 19) - (2.22) refer to the inertial frame $\Sigma(r, t, w)$ with substratum flow $w$ As before, the Galilei covariance of these equations could be proven by applying to them the Galilei transformations (2 23) - (2.28). However, the Galilei covariance of Eqs. (3.1) - (3.3) and Eqs (2 19) - (222) is more easily established by means of the covariants and invariants of the Galilei transformation $\Sigma(\mathbf{r}, \mathrm{t}, \mathbf{w}) \rightarrow \Sigma^{\prime}\left(\mathbf{r}^{\prime}, \mathbf{t}^{\prime}, \mathbf{w}^{\prime}\right)$ :

$$
\begin{gathered}
\partial / \partial \mathrm{t}+\mathbf{v} \cdot \boldsymbol{\nabla}=\partial / \partial \mathrm{t}^{\prime}+\mathbf{v}^{\prime} \cdot \boldsymbol{\nabla}^{\prime}, \quad \boldsymbol{\nabla}=\boldsymbol{\nabla}^{\prime}, \\
\mathbf{E}+\mathbf{v} \times \mathbf{B}=\mathbf{E}^{\prime}+\mathbf{v}^{\prime} \times \mathbf{B}^{\prime}, \\
\rho=\rho^{\prime} \quad . \quad \mathrm{L}=\mathrm{L}^{\prime},
\end{gathered}
$$

and

$$
\begin{aligned}
& \partial / \partial t+\mathbf{w} \cdot \boldsymbol{\nabla}=\partial / \partial \mathrm{t}^{\prime}+\mathbf{w}^{\prime} \cdot \boldsymbol{\nabla}^{\prime}, \boldsymbol{\nabla}=\boldsymbol{\nabla}^{\prime} \\
& \boldsymbol{\Phi}-\mathbf{w} \cdot \mathbf{A}=\boldsymbol{\Phi}^{\prime}-\mathbf{w}^{\prime} \cdot \mathbf{A}^{\prime}, \mathbf{A}=\mathbf{A}^{\prime}, \\
& \mathbf{j}_{e}-\rho_{e} \mathbf{w}=j_{e^{\prime}-\rho_{e} \mathbf{w}^{\prime},}, \rho_{e}=\rho_{e}^{\prime},
\end{aligned}
$$

where

$$
\mathbf{v}=\mathbf{v}^{\prime}+\mathbf{u}
$$

which implies $\nabla \cdot v=\nabla^{\prime} \cdot v^{\prime}$ and $\nabla \mathbf{v}=\nabla^{\prime} v^{\prime}$. Equations $(36)-(3.11)$ follow immediately from the Galilei transformations $(2.23)-(2.28)$ Now it is seen directly that (i) the hydrodynamic quantum equations (3.1) and (3) are Galilei covariant by Eqs. (3.6) - (3.8) and Eq. (3 12) and (ii) the associated electromagnetic equations (2 19) - (2.22) are Galilei covariant by Eqs. (3.9) - (3.11)

It is interesting to investigate the Galilei covariance of the quantization of the circulation of the canonical momentum $m_{0} v+e_{0} A$. By Eqs. (3.6), (3.10), and (3.12),

$$
\oint\left(m_{0} \mathbf{v}^{\prime}+e_{0} \mathbf{A}^{\prime}\right) \cdot d \mathbf{r}^{\prime}=\hbar 2 \pi m, \quad m=0, \pm 1, \pm 2, \quad \pm \infty,
$$

since $\oint u \cdot d r^{\prime}=0$. Accordingly, the circulation of the canonical momentum is a Galilei invariant.

The Galilei transformation of the hydrodynamic equations $(31)-(32)$ of quantum mechanics is mathematically simple in comparison with the Galilei transformation of the Schroedinger equation (2 18), as is evident from its operator $\left.\left(-i \hbar \nabla-e_{1}\right) A\right)^{2}$ which acts on the wave function $(225)$. Equations $\left(\begin{array}{l}31 \\ 1\end{array}\right)-(32)$ have static solutions with $\partial / \partial \mathrm{t}=\mathbf{0}$ and $\mathbf{v}=\mathbf{0}(\mathrm{e} . \mathrm{g}, \mathrm{m}=0$ states of $\mathrm{H}$-atom) and dynamic solutions with $\partial / \partial \mathrm{t}=0$ and $\mathbf{v} \pm 0$ le $\mathrm{g}, \mathrm{m} \neq 0$ states of $\mathrm{H}$-atom), which have been discussed previously (Wilhelm $[25\}$ ). The solutions of the Schroedinger equation (2.18) with $\partial / \partial t \equiv 0$ appear to have no physical meaning

\section{CONCLUSION}

A consistent, Galilei covariant representation of the quantum mechanics of a charged particle in electromagnetic fields has been achieved The Galilei transformations for the wave and hydrodynamic fields of quantum mechanics and the electromagnetic fields have been derived from first principles Thus, the formal 
difficulties resulting from the mixing of Galilei and Lorentz covariant field equations in "nonrelativistic" quantum mechanics have been removed.

The Schroedinger and electromagnetic wave equations (2.18) - (22) with substratum and their Galilei transformations (2.23) - (2.28) represent a field theory in terms of absolute or Galilean space $r=(x, y, z)$ and time $t$ coordinates (i.e., the Galilean coordinate differences are the same in all inertial frames, $\Delta \mathbf{r}=$ in $v$ and $\Delta t=i n v)$. According to Lorentz [28], a measuring rod resting parallel to the ether velocity $w$ in an inertial frame $\Sigma(r, t, w)$ has there the reduced length $L(w)=L_{0}\left(1-w^{2} / c^{2}\right)^{\frac{1}{2}}$ due to the flattening of its microscopic Coulomb fields by the ether flow $\left(L_{0}=\right.$ proper rod length in the ether rest frame $\left.\Sigma_{0}\right)$. Recognizing that a clock can be visualized as a system reflecting a light signal back and forth between two mirrors held apart by a rod. Builder $[29,30]$ demonstrated that the period of a clock is increased to $T(w)=T_{0} /\left(1-w^{2} / c^{2}\right)^{\frac{1}{2}}$ in the inertial frame $\Sigma(r, t, w)$ with ether flow $w$, as the combined result of the rod contraction and the anisotropy of light propagation between the mirrors $\left(\mathrm{T}_{0}=\right.$ proper clock period in the ether rest frame $\left.\boldsymbol{\Sigma}_{\boldsymbol{n}}\right)$. For these physical reasons, the differences $\Delta$ of the absolute Galilean coordinates $r, t$ of the inertial frame $\Sigma(r, t, w)$ are related to the measured (m) space and time coordinate differences in this reference frame by (\| and $\perp$ to $w$ )

$$
\Delta \mathbf{r}_{m}^{\prime}=\mathrm{Y}^{\prime} \Delta \mathbf{r}^{\prime}, \Delta \mathbf{r}_{m^{\perp}}=\Delta \mathbf{r}^{\perp}, \Delta t_{m}=\Delta t / Y,
$$

where

$$
Y=\left(1-w^{2} / c^{2}\right)^{\frac{1}{2}}, 0 \leq|w|<c
$$

In general, the measured coordinate differences $\Delta r_{m}{ }^{\prime}$ and $\Delta t_{m}$ vary in accordance with the $\gamma(w)$ of the respective inertial frame $\boldsymbol{\Sigma}$. This is in agreement with the Michelson-Morley and Doppler experiments (Whittaker [34]) Since the scaling factor $Y(w)$ is a constant for a given inertial frame $\Sigma(r, t, w)$, the calculation of the measured ( $\mathrm{m}$ ) coordinates from the Galilean coordinates, and vice versa, by means of Eqs. (4.1) - (4.2) is elementary in applications.

Thus, Eqs. (4.1) - (4.2) permit to take into account the principal relativistic effects. namely length contraction and time dilatation, in Schroedinger's quantum mechanics. Length contraction and time dilatation are of great importance for high velocity phenomena. For proper nonrelativistic quantum mechanics and electromagnetic theory, length contraction and time dilatation are quantitatively negligible effects since $Y=1$ for $|w| \ll c$. In this case, the reinterpretation of the space and time coordinates of incrtial frames with substratum flow is unnecessary

\section{REFERENCES}

1. LANDAU, L. D. and LIFSHITZ, E. M. Quantum Mechanics, Pergamon, New York, 1971.

2. WILhel.M, H E and HONG, S. H. Anomalous Propagation of Probability in Quantum Mechanics, $\underline{\text { Acta }}$ Phys. Hung. $\underline{48}(1980), 425-437$.

3. BARLT, A. O. The Schroedinger Equation - 50 Years Later, Z. Naturforschung 32a (1977), 362-374

4. HLSL:MI, K. Miscellanea in Elementary Quantum Mechanics, Prog. Theor. Phys. 2 (1953), $381-402$

5. STLTZ, C. and SCHLITT, D. W. Temporal Evolution and Approach to Equilibrium of a Quantum Particle in a Suddenly Expanded Box, Phys. Rev. A2 (1970), 897-917.

6. WILHELM, H. E. Wavefunction, Energy Spectrum, and Cncertainty Relation for a Particle Contained Between Moving Potential Walls, J. Phys. A16 (1983), 2149-2159.

7. POINCARÉ, H. Lorentz Theory and Reaction Principle, Arch. Neerl. Sci. $\quad \underline{5}(1900), 252-278$.

8. HASENOEHRL, F. Report on the Inertia of Energy, Jahrb. Radioaktivitaet $\underline{6}$ (1909), 485-502.

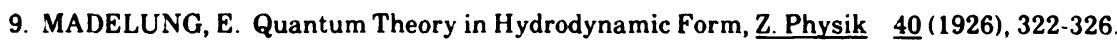

10. WILSON, R. W. A History of the Discovery of the Cosmic Microwave Background Radiation, Phvs Scr 21 , (1980), 599-604

11. MANSOLRI, R. and SEXL, R. L. A Test of Special Theory of Relativity Simultaneity and Clock Synchronization, Gen. Rel. Grav. 8 (1977), 497-513. 
12. BAGGE, E. What is Inertia? Atomkernenergie $\underline{34}$ (1979), 30-34.

13. BAGGE, E. Quantum-Mechanics of Spatially Extended Elementary Particles, Naturwissenschaften $\underline{23}$ (1962), 1.

14. WINTERBERG, F. Nonlinear Generalization of Special Relativity at Very High Energies, Atomkernenergie 44 (1984), 238-246.

15 A.NDERSON, C. D. The Positive Electron, Phys Rev. $\underline{43}$ (1933), $491-494$

16 DIRAC, P A. M. The Principles of Quantum Mechanics, Clarendon, Oxford, 1958

17. POWER, E. A. Introductory Quantum Electrodynamics. Elsevier, . New York, 1964

18 I.ANDAL' L. D. and LIFSHITZ, E .11 Classical Theorv of Fields, Pergamon, Oxford, 1970

19 WHEELER, J A. Geometrodynamics, Academic, . New York, 1962.

20 WINTERBERG, F. Non-Einsteinian Relativity at High Energies, Unpublished, 1984.

21. EI.NSTEI.N, A. Geometry and Experience, Preuss Akad Wissensch . Verh. (1921), 123-130

22 STRATTON, J A. Electromagnetic Theory, McGraw-Hill, New York, 1941

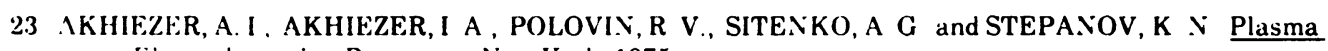
Electrodynamics, Pergamon, .lew York, 1975.

24 WHIT'TAKER. A Historv of the Theories of Ether and Electricity, Longmans, London, 1910

25 WII.HEI.U, II E Hydrodynamic Model of Quantum Mechanics, Phvs Rev D1 (1970), 2278-2285.

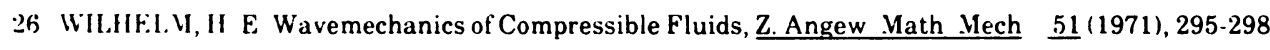

27 WII.HEI. I. H E Wave-.Mechanical Formulation of Many-Fluid Plasma Thenry in Electromagnetic Fields, Phis Rev 3A (1971), 399-404

28 I.ORE.NT\%, H. A. The Theorv of Electrons, Teubner, Leipzig, 1909

29 BLILDER, G. Ether and Relativity, Aust. J. Phvs. 11 (1958), 279-297

30 BLIL.DER, G. The Constancy of the Velocity of Light, Aust. J. Phvs. 11 (1958), 457-480 


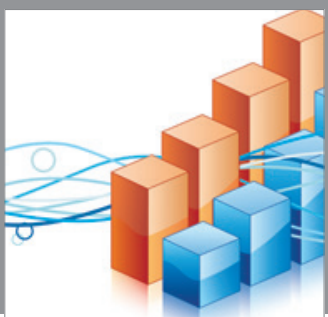

Advances in

Operations Research

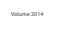

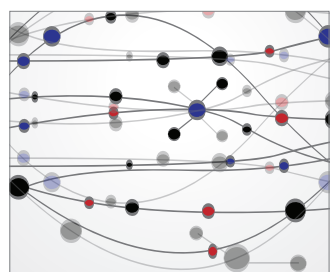

\section{The Scientific} World Journal
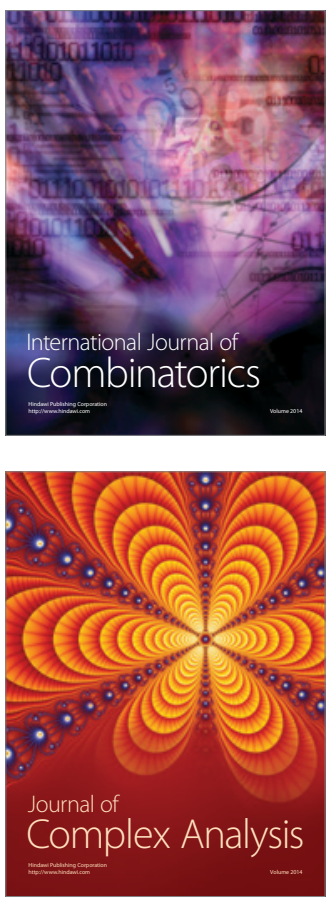

International Journal of

Mathematics and

Mathematical

Sciences
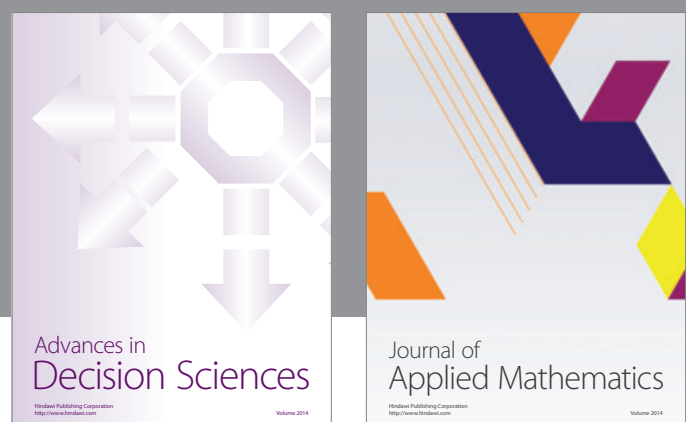

Journal of

Applied Mathematics
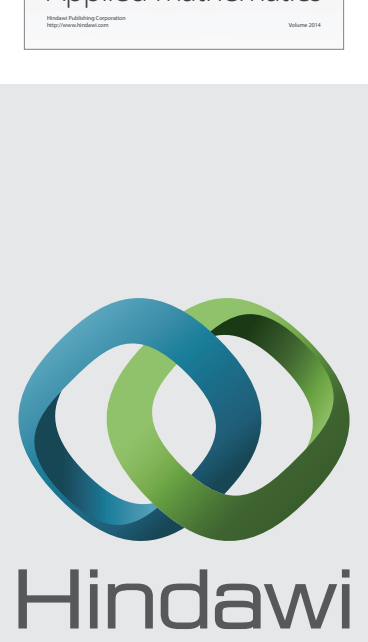

Submit your manuscripts at http://www.hindawi.com
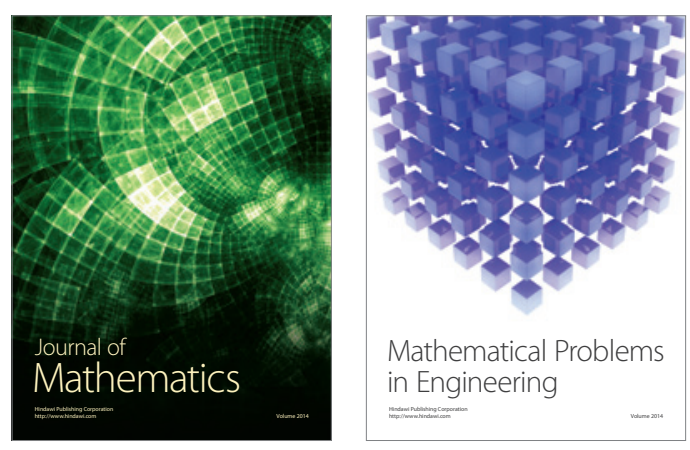

Mathematical Problems in Engineering
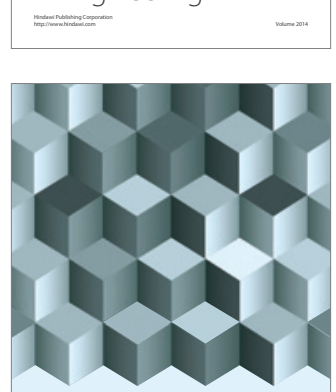

Journal of

Function Spaces
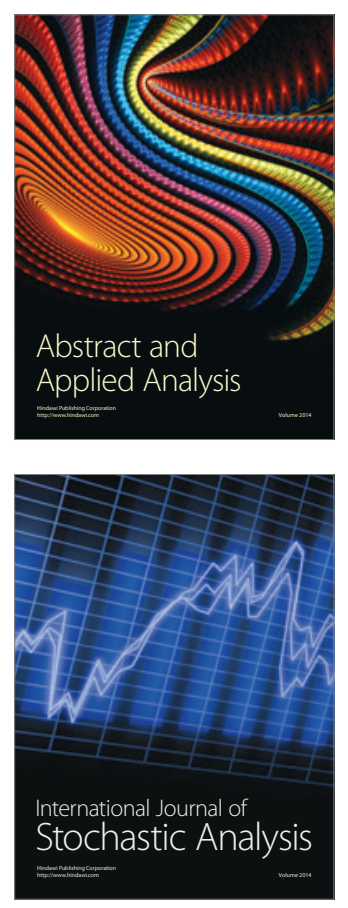

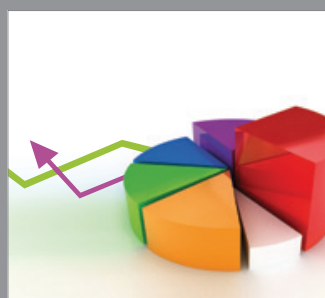

ournal of

Probability and Statistics

Promensencen
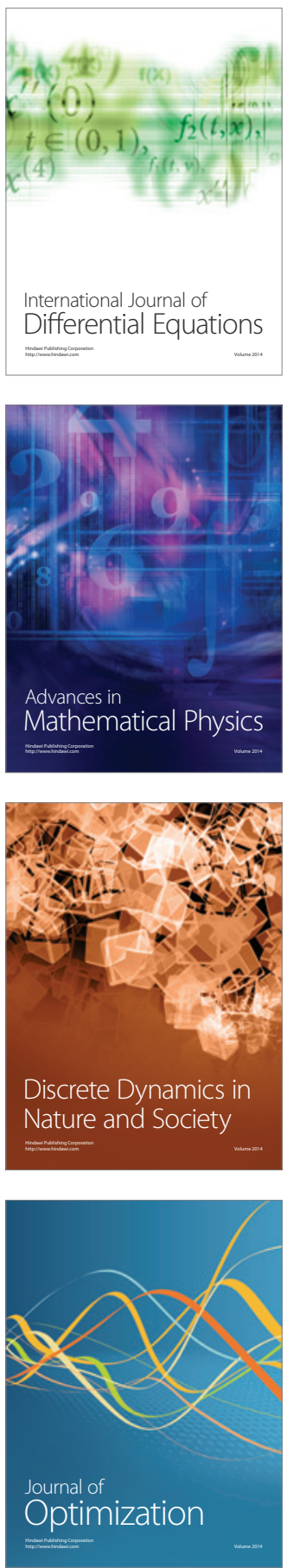